\title{
Review Article \\ Rotordynamic Faults: Recent Advances in Diagnosis and Prognosis
}

\author{
Ryan Walker, Sureshkumar Perinpanayagam, and Ian K. Jennions \\ Integrated Vehicle Health Management Centre, University of Cranfield, Bedfordshire MK43 OAL, UK \\ Correspondence should be addressed to Ryan Walker; r.b.walker@cranfield.ac.uk
}

Received 8 October 2012; Revised 15 January 2013; Accepted 21 January 2013

Academic Editor: Arthur Lees

Copyright (C) 2013 Ryan Walker et al. This is an open access article distributed under the Creative Commons Attribution License, which permits unrestricted use, distribution, and reproduction in any medium, provided the original work is properly cited.

\begin{abstract}
Diagnosis and condition monitoring in rotating machinery has been a subject of intense research for the last century. Recent developments indicate the drive towards integration of diagnosis and prognosis algorithms in future integrated vehicle health management (IVHM) systems. With this in mind, this paper concentrates on highlighting some of the latest research on common faults in rotating machines. Eight key faults have been described; the selected faults include unbalance, misalignment, rub/looseness, fluid-induced instability, bearing failure, shaft cracks, blade cracks, and shaft bow. Each of these faults has been detailed with regard to sensors, fault identification techniques, localization, prognosis, and modeling. The intent of the paper is to highlight the latest technologies pioneering the drive towards next-generation IVHM systems for rotating machinery.
\end{abstract}

\section{Introduction}

The topic of diagnosing and prognosing faults in rotating machinery is an ongoing subject of research, with many developments published in a range of conferences and journals annually. This research has the potential to become even more relevant in the coming years due to the rise of IVHM, in which the drive towards condition-based maintenance and whole vehicle monitoring plays a vital role. This paper intends to survey some of the recent developments in the field, with the aim of summarizing some of the more promising studies and trends with relevance to future IVHM systems for rotating machinery.

Modern day rotating machines operate with a high level of reliability, and yet the drive for ever increased operation and decreased unscheduled maintenance is providing additional challenges for industry. The airline industry provides a current example of this desire, with airlines pushing manufacturers to enable shorter turnaround times and to keep aircraft in the air longer, increasing cost benefit. Despite the high level of reliability, the rotordynamic faults detailed in this paper remain aspects which require consideration in this drive for increased reliability and improved maintenance procedures [1].
In order to fully understand and summarize the trends and developments in this area, several hundred recent conference and journal papers have been studied. Overall trends have been highlighted and discussed alongside specific papers of relevance. It is intended that the work should provide a broad reference and summary for working engineers on some of the latest developments in rotordynamic fault diagnosis and prognosis, with specific application to papers of industrial relevance for the drive towards future IVHM systems.

In order to fully study the diagnosis and prognosis of rotordynamic faults, it has been deemed necessary to break down the topic of rotordynamic faults into sections defined as follows:

Sensors: sensors commonly used for diagnosis of specific faults.

Fault Identification: diagnosis and root cause detection.

Localisation: locating a specific fault within a complex system.

Prognosis: prognosis of components and remaining useful life.

Modelling: simulation of rotordynamic faults. 
Through the study of the topics listed, it is useful to place the research conducted in this paper into context with regard to real-world applications. Further to this, it is intended to identify potential areas where more research is required in order to push some of the recent technologies highlighted for this study into industry.

Anderson [2] provides a summary of maintenance time breakdown for a collection of military aircraft. This indicates that as much as $44 \%$ of on-aircraft maintenance time (which in turn accounts for $90 \%$ of total maintenance operations) is consumed with inspection alone. The techniques addressed in this paper enable the maintenance to be more informed and targeted, with inventory ready when needed, providing a significant contribution to reducing maintenance time and cost.

As the topic of rotordynamic faults is very large area of research, the scope of this paper has to be limited. The choice of faults has been made after considering the works by Muszynska [3] and Bently [4], both of whom consider the fundamentals of common faults in much detail. Out of the wide range of possible rotordynamic faults, eight have therefore been selected. Due to the general reliability of the current generation of gas turbines, faults falling outside of the eight listed have been classed as "uncommon" for the purposes of this study. This decision was made by assessing the severity of each fault, dependences on other faults, and the level of research dedicated to diagnosis of each fault.

The scope of this paper is therefore confined to the following faults:

(i) unbalance,

(ii) misalignment,

(iii) rub and looseness,

(iv) fluid-induced instability,

(v) bearing faults,

(vi) shaft cracks,

(vii) blade cracks,

(viii) rotor bow.

Each of these faults is varied, and some are more common than others. The consensus from the works reviewed within this paper is that unbalance is the most commonly occurring, in part due to the fine tolerances applied to modern machinery and also due to the links between unbalance and other faults. Misalignment can also be identified as another common fault. The particular importance of these two faults is highlighted by Domes [5] who discusses common faults from the perspective of Rolls Royce. Bearing failure is perhaps the most diverse fault here and has the highest concentration of research in various areas. The decision has also been made to separate faults occurring on the shafts of a system to those occurring on rotors, as these can be classified as distinct faults-particularly with regards to localization and maintenance. Despite these conclusions, quantifying the faults with regards to the rate of occurrence was not possible due to the lack of commercially available data, and each of the eight faults has therefore been investigated to an equal level of detail in this paper.
It should be noted that although the faults have been separated into eight categories they are by no means mutually exclusive. Dependencies exist between many of the faults. A common example of this is the interaction between unbalance and misalignment (outlined by Bently [4]). Fault chains can become even more complex, misalignments leading to an unbalance which causes a rub for an example. Some works contained herein detail single fault analysis, and a few refer to two or more faults. Such fault dependencies are one of the limiting factors when moving technologies from the lab into industry. An example of this would be a system capable of diagnosing unbalance faults, without consideration for misalignment as a root cause may render such a system ineffective when applied to a real-world scenario.

The selected papers have been further refined based upon relevance to aeroengine gas turbines and publication date (with works from the last three years preferred).

As research into the diagnosis and prognosis of faults in rotating machines is a developing topic, the application of such technologies in industry has not yet reached a stage where there are common solutions, or even a set of established procedures to follow. This holds particularly true with regards to the broader fields of IVHM. Taking the state-ofthe-art Joint Strike Fighter (JSF) program as one example of the current level of technology in circulation, although the autonomic logistics proposed by Hess et al. [6] for the vehicle is undeniably impressive, it is still a long way from implementing some of the recent technologies claimed to be successful in a lab-based environment.

Although, as mentioned, an established and commonly applied set of procedures and standards for IVHM has not yet emerged, with regards to rotating machinery a number of standards and procedures can be referred to with regards to detailing and quantifying the faults. These include such aspects as covered by ISO 2953:1999, which details correct balancing procedures and levels of mechanical vibration. Another example would be in the UK Ministry of DefenceMilitary Aviation Authority (2010), JAP 100A-01-Military aviation engineering policy and regulation, where a mention is made of procedures for debris and vibration monitoring. This indicates a gradual move towards common ground for procedures and standards for IVHM systems. Despite this research remains varied, and separating the research with potential for moving beyond the lab-based environment into industry can be difficult to identify at first glance.

The discussion contained herein therefore takes into account the above detailed considerations and constraints, with the aims of the paper as follows.

(i) Highlight key examples of the latest research in eight common rotordynamic faults.

(ii) Summarise current trends identified from the study.

(iii) Detail the current state of the art and the future work required for next generation IVHM systems for rotating machinery.

\section{Rotordynamic Faults}

The following section details the aforementioned common rotordynamic faults with regard to recent physics-based 
simulation work and traditional data-driven methods. Research in this area of rotordynamics is particularly widespread, and so this paper outlines only a few recent areas of research. Although not being of mainstream interest, a list of commonly used sensors has been included. Table 1 represents a summary of the information collected and detailed as a part of this paper.

As discussed, a wide selection of papers have been reviewed for consideration in this paper, of which it is not possible to describe them all in detail. In an attempt to highlight some of the common themes across many of works reviewed, Table 1 has been created. Table 1 by no means details all relevant methods and approaches currently being investigated; however, it details themes which were found to be common across many areas of current research. It is intended for the detailed table to compliment the important works described in more detail in the following section of the paper, separated by fault type.

2.1. Unbalance. This is one of the most common rotordynamic faults [4]; every rotating machine has an inherent degree of unbalance. Unbalance as a fault can, therefore, be defined as unbalance outside of a given tolerance level. A recent piece of research which demonstrates the ongoing development of data-driven techniques is that by Ganeriwala et al. [7], who tested a technique for measuring operating deflection shapes (ODS) in order to detect unbalance cases. These studies were conducted on a machine fault simulator (a physical simulator of common faults in rotating machines) such simulators have the advantage of recreating faulty conditions quickly and easily, enabling a new dimension to datadriven diagnostic techniques. It is, however, worth mentioning that data for these experiments were collected using 14 accelerometers, which are easy to apply to such a simulator, but it may be much more difficult to configure these many sensors on a complex system. Despite this, the paper achieved its aim in proving the hypothesis "when an operating machine becomes unbalanced, its ODS will change."

Regarding physics-based simulation of unbalance as a fault, the work by Sudhakar and Sekhar [8] described a model-based method for fault identification using a minimal sensor suite. This is achieved through the analysis of transverse vibrations at a single location. Throughout the paper, three different approaches are studied-least squares minimization, equivalent loads minimization, and vibration minimization. A reduced error is found to occur using a proposed modification to the typical equivalent loads minimization approach. The work is of note due to the authors requirement to both identify and locate (localize) unbalance.

Localization and prognosis of unbalance pose a unique set of challenges and as a result, research is still somewhat limited in these areas. One recent work which claims to localise unbalance accurately is by Yang and Hsu [9]; the authors use trending data and reasoning systems to locate unbalance and shaft bow across a system. Quick diagnosis is achieved by avoiding the study of all fault combinations, and the authors claim the ability to localise shaft bow and unbalance in large rotating machines. The techniques rely extensively on previous searches and are limited to large, stable machines operating at a specific RPM. Remaining useful life of unbalance is difficult to predict due to complicating factors. As discussed, the fault dependencies are such that in many cases the unbalance itself will not be the failing factor, instead an unbalance may cause a rub which then leads to damage which can be prognoses. Such combinations of faults and underlying causes for unbalance lead to the need for remaining useful life predictions to be made based upon the exact nature of a specific fault.

2.2. Misalignment. This is another common fault which can potentially inflict considerable damage in rotating machines. As with unbalance, misalignment in a whole system can be complicated by secondary faults (e.g., a misalignment which causes a rub). El-Shafei et al. [10] are an example of the ongoing research in this area; in this case, a unique combination of angular misalignment and oil whip/whirl is detailed. The authors describe how small degrees of misalignment can be utilized in order to prevent the onset of fluid-induced instabilities, tested through the use of a lab-based test rig. Such research presents a new dimension in looking at common rotordynamic faults, with aspects that could be applied to future design for IVHM systems.

El-Shafei et al. [11] demonstrate interesting research into misalignment from the perspective of physics-based simulation. The authors construct mathematical models of a simple rotor system with a misaligned coupling and collect harmonic response data from this to assess the severity of different misalignment cases. Such models are useful throughout the life of rotating machines-from design to implementation, although again successful validation with experimentally obtained data is key. The authors highlight the fact that although misalignment is a prevalent and serious fault, no comprehensive research has been performed for treating this problem. The methodology applied includes deriving the energy expressions applying the Ritz series method, constructing the equations of motion and then using the harmonic balance method to look for multiharmonic responses. The paper demonstrates the ongoing research to understand and model the fundamentals of such faults, in order that improved diagnosis and prognosis methods can be built upon such knowledge.

As with unbalance, localization and prognosis of misalignment is a complex topic to research. Studies such as ElShafei et al. [11] can make accurate predictions for misalignment in a simple system with one coupling. However, real systems (e.g., aircraft gas turbines) have many potential locations of misalignment. This is an area where few researchers have made an impact. Remaining useful life predictions for misalignment is complicated for the same reasons as with unbalance. Villa et al. [12] discuss statistical diagnosis of misalignment faults, with reference to prognosis. The authors use the example of a wind turbine for their studies, but stress the applicability to other systems. Differentiation with unbalance faults is also covered (as these two faults are closely linked). Unlike the work by El-Shafei et al. [11], emphasis is given to the machine in question operating over a wide range of operating speeds and conditions. This is achieved through the use of an angular resampling method. Prognosis is tackled 
TABLE 1: Rotordynamic "fault table."

\begin{tabular}{|c|c|c|c|c|c|}
\hline Faults & Sensors & $\begin{array}{l}\text { Fault identification } \\
\text { techniques }\end{array}$ & Localisation of fault & Prognosis & $\begin{array}{l}\text { Work of note in fault } \\
\text { modelling }\end{array}$ \\
\hline Unbalance & $\begin{array}{c}\text { Accelerometer } \\
\text { Velocity transducer } \\
\text { Proximity transducer }\end{array}$ & $\begin{array}{l}\text { Operating deflection } \\
\text { shapes } \\
\text { Time-frequency } \\
\text { analysis and many } \\
\text { others }\end{array}$ & $\begin{array}{l}\text { Neural-network } \\
\text { modelling and } \\
\text { time-frequency } \\
\text { analysis }\end{array}$ & FCM-Markov model & $\begin{array}{l}\text { Unbalance in full-engine } \\
\text { FEA model, } \\
\text { Early detection in } \\
\text { aeroengines }\end{array}$ \\
\hline $\begin{array}{l}\text { High radial } \\
\text { loads and } \\
\text { misalignment }\end{array}$ & $\begin{array}{l}\text { Accelerometer } \\
\text { Thermocouple } \\
\text { Displacement } \\
\text { transducer } \\
\text { Angular sensor }\end{array}$ & $\begin{array}{l}\text { Bearing temperature } \\
\text { observation advanced } \\
\text { Software-driven } \\
\text { filtering methods }\end{array}$ & $\begin{array}{l}\text { Various vibration } \\
\text { analysis techniques }\end{array}$ & $\begin{array}{l}\text { Prognostic } \\
\text { modelling using } \\
\text { simulated vibration } \\
\text { data }\end{array}$ & $\begin{array}{l}\text { Modelling the } \\
\text { relationship between } \\
\text { misalignment and other } \\
\text { faults } \\
\text { Harmonic balance } \\
\text { method }\end{array}$ \\
\hline $\begin{array}{l}\text { Rub and } \\
\text { looseness }\end{array}$ & $\begin{array}{l}\text { Accelerometer } \\
\text { Velocity transducer } \\
\text { Optical sensor }\end{array}$ & $\begin{array}{l}\text { Single value } \\
\text { decomposition } \\
\text { Time-frequency } \\
\text { analysis }\end{array}$ & $\begin{array}{l}\text { Least-squares fitting } \\
\text { approach }\end{array}$ & $\begin{array}{l}\text { Adaptive wavelet } \\
\text { denoising for } \\
\text { prognostics }\end{array}$ & $\begin{array}{l}\text { Rob and looseness using } \\
\text { FEA models Whole } \\
\text { engine modelling } \\
\text { techniques }\end{array}$ \\
\hline $\begin{array}{l}\text { Fluid-induced } \\
\text { instability }\end{array}$ & $\begin{array}{l}\text { Proximity probes } \\
\text { Keyphase transducer }\end{array}$ & $\begin{array}{l}\text { Full spectrum plots } \\
\text { Large amplitude } \\
\text { subsynchronous } \\
\text { vibration } \\
\text { Detection }\end{array}$ & $\begin{array}{l}\text { Blade tip clearance } \\
\text { and loading } \\
\text { distribution }\end{array}$ & $\begin{array}{l}\text { Cross-coupled } \\
\text { stiffness and } \\
\text { direct-damping } \\
\text { studies }\end{array}$ & $\begin{array}{l}\text { Nonlinear modelling } \\
\text { accuracy of model based } \\
\text { Diagnostics numerical } \\
\text { modelling }\end{array}$ \\
\hline Bearing failure & $\begin{array}{l}\text { Accelerometer } \\
\text { Velocity transducer } \\
\text { Displacement } \\
\text { transducer } \\
\text { Proximity probes }\end{array}$ & $\begin{array}{l}\text { Postfailure analysis for } \\
\text { future failure diagnosis } \\
\text { Dynamic } \\
\text { time-frequency analysis }\end{array}$ & $\begin{array}{l}\text { Statistical change in } \\
\text { the bispectral } \\
\text { domain }\end{array}$ & $\begin{array}{l}\text { Numerical } \\
\text { modelling of } \\
\text { aeroengine bearings } \\
\text { Model-based } \\
\text { prediction of } \\
\text { remaining useful life } \\
\text { On-board } \\
\text { prognostics }\end{array}$ & $\begin{array}{l}\text { High and low fidelity } \\
\text { dynamic system } \\
\text { modelling } \\
\text { Comparisons of } \\
\text { algorithms to } \\
\text { understand bearing } \\
\text { vibration phenomena }\end{array}$ \\
\hline Shaft cracks & $\begin{array}{l}\text { Keyphase transducer } \\
\text { Proximity probes }\end{array}$ & $\begin{array}{l}\text { Higher order spectra } \\
\text { Neural networks } \\
\text { Grid support vectors }\end{array}$ & $\begin{array}{l}\text { Forced response } \\
\text { measurements }\end{array}$ & $\begin{array}{l}\text { Nonintrusive } \\
\text { torsion vibration } \\
\text { monitoring }\end{array}$ & $\begin{array}{l}\text { Statistical analysis FEA } \\
\text { modelling }\end{array}$ \\
\hline Blade cracks & $\begin{array}{l}\text { Keyphase transducer } \\
\text { Proximity probes }\end{array}$ & $\begin{array}{l}\text { Hilbert-Huang } \\
\text { transformation } \\
\text { Model-based } \\
\text { diagnostics } \\
\text { Morlet } \\
\text { wavelet/quantum } \\
\text { techniques }\end{array}$ & $\begin{array}{l}\text { Novel bearing } \\
\text { structures }\end{array}$ & $\begin{array}{l}\text { Active magnetic } \\
\text { actuator }\end{array}$ & $\begin{array}{l}\text { Global and local } \\
\text { asymmetry modelling } \\
\text { Finite element modelling }\end{array}$ \\
\hline Rotor Bow & $\begin{array}{l}\text { Keyphase transducer } \\
\text { Proximity probes } \\
\text { Seismic transducer }\end{array}$ & $\begin{array}{l}\text { Examination of "slow } \\
\text { roll" data } \\
\text { Model-based } \\
\text { diagnostics }\end{array}$ & $\begin{array}{l}\text { Partial mathematical } \\
\text { modelling }\end{array}$ & $\begin{array}{l}\text { Statistical symptoms } \\
\text { analysis }\end{array}$ & $\begin{array}{l}\text { Advantages of fault } \\
\text { modelling for } \\
\text { identification } \\
\text { Nonlinear modelling } \\
\text { techniques }\end{array}$ \\
\hline
\end{tabular}

through the use of a statistical diagnosis algorithm based on the significance level of the faults in question.

2.3. Rub and Looseness. Rub is always a secondary fault (i.e., a product of another fault such as looseness) and can lead to fatigue and wear. Rub and looseness can create complex vibration signals which are difficult to diagnose using traditional methods. Modeling and simulation of rub and looseness faults have been considered in several recent works.

This includes Ngolah et al. [13], which detail the monitoring and diagnosis of common faults (including rub and looseness) based upon a three-layer Artificial Neural Network (ANN). A series of 10 key performance indicators were identified and used as training. The authors test the system in a lab environment, but stress the applicability to industrial applications. The research indicates one of the latest methods of research which enables the implementation of diagnosis techniques. It is a useful tool for rub and looseness studies, as it incorporates a variety of faults which could "underlie" such a fault. Despite this, the research relies on clear features for each fault, which can be much easier to identify in a lab environment as opposed to "noisy" industrial applications. 
Lu et al. [14] have performed several studies into rub and looseness, including this example in which a flexibly mounted shaft has an induced rub due to a range of contact rings. The study focusses on the potentially dangerous effects of rub in causing excessive nonsynchronous and chaotic vibrations. The links with unbalance and misalignment are discussed and detailed. The considerations for real-world cases in the described research are considerable, as part of the drive towards full understanding of the nonlinear effects of rub and looseness.

Localization of rub and looseness across whole systems is relatively lightly studied in the literature. Many works (including those already cited) look at single or dual-rotor systems where localization of such faults is not an issue. In an industrial setting, complex systems may comprise many rotors in several compressor and turbine stages, significantly complicating diagnosis of such faults. Research into prognosis of rotor-stator rubs lies mostly within the domain of datadriven techniques. Modeling and simulation research can be used to support data-driven techniques for prognosis and condition-based monitoring. Han et al. [15] is an example of this; the authors use finite element modeling to construct a dual rotor model. Various types of rub-impact are then studied. Such studies can provide a wide range of information, which can then be combined with data obtained from live systems, potentially with seeded faults, in order to construct accurate remaining useful life predictions. As pointed out by the authors, one key advantage of simulation is the ability to study more complex systems with a higher number of rotors, which is used throughout this research. This presents a different approach to identifying features for identification of rub and looseness.

2.4. Fluid-Induced Instability. Fluid-induced instabilities (often referred to as whip and whirl) are potentially very serious faults which can result in wear, fatigue, and extensive damage to machine components. Such instabilities can be found in interstage seals, fluid lubricated bearings, and blade-tip clearances. Research into simulating and modeling fluid-induced instability has produced several works of interest to fault diagnosis of rotating machines in the last few years. De Castro et al. [16] are a good example, where nonlinear mathematical models are prepared for a rotorbearing system. The models are then used to predict instability thresholds. The authors consider a test case against a power plant turbine and a test rig, therefore validating the simulations. The case of unbalance faults causing whip and whirl phenomena is also considered. The main conclusion therefore drawn from the work is that the authors concluded that nonlinear hydrodynamic journal bearing models enable sufficiently accurate simulations for predicting instability thresholds. Fan et al. [17] represent an example of work from the perspective of aeroengine turbines. In this case, startup conditions are studied using a full Hilbert spectrum. The aim behind the paper is to accurately predict the point at which whip and whirl occur, thus enabling this to be avoided at the design stage. Such findings could potentially also be used to identify whip and whirl as the case of a fault after a period of wear in the operating machine.
Prognosing fluid-induced instability is a relatively lightly researched topic. Fluid instabilities can be covered as part of extensive research into remaining useful life of bearings. The potential exists for modeling and simulation techniques such as those detailed above to become a part of prognosis for fluid-induced instabilities due to the fact that it can be very difficult to seed such faults into live systems for testing and evaluation. As with other faults detailed in this paper, many studies have been performed with the aim of describing fluidinduced instabilities based on the measurement or simulation of single (or occasionally dual) rotor setups. Physics-based simulation with the aim of localising fluid instability faults across a whole system can be limited by the complexity of both the fault and the system, hence, the simplification to single rotor-stator bearing systems.

2.5. Bearing Failure. An area where data-driven techniques are still providing the basis of much research in the field of rotordynamics is that of bearing failure. The title "bearing failure" can cover a wide range of potential issues which continue to be studied in detail. Faults can occur in all kinds of engine bearings-the inner and outer case, the cage and the rolling elements, fluid-induced instabilities (addressed in a separate section), lubrication, and the complexities of active magnetic bearings to name some examples. All types of bearing relevant to rotating machinery are the subject of ongoing research, and this subject has the potential to form several separate papers. As a brief highlight, some recent examples are discussed as follows.

Data-driven techniques have enabled accurate bearing diagnostics and prognostics to be described for a range of rotordynamic systems. Despite the prevalence of datadriven research in this area, research from a physics-based simulation perspective has also recently produced some interesting papers of relevance to condition monitoring and health management of rotating machinery.

This includes Kappaganthu and Nataraj [18], in which rolling element bearings have been studied through the use of nonlinear models. The included nonlinearity in this case is clearance, and the model is then used in order to study chaotic motions, in particular the regions of chaotic response. The research forms a part of an ongoing drive to develop an accurate model-based diagnostic technique for rolling element bearings, taking into account clearance nonlinearities and chaotic responses.

Gupta et al. [19] demonstrate another example of the latest research into instability and chaos in rolling element bearings through high-fidelity simulations. This detailed and complex study involves the application of a novel scheme to analyze the quasiperiodic response of the system combined with a nonautonomous "shooting" method. This work highlights the level of detail to which nonlinearities and complex nonlinear motions in bearings are beginning to be understood and accurately modeled. Again, such work presents the potential for design of IVHM in future evolutions of the research.

As so much research has been performed (and is ongoing) into bearing faults across a wide variety of mechanical systems, both prognostics and localization of bearing faults 
have been researched in somewhat more detail than some of the other faults detailed here. Despite this, much work still needs to be performed in order to translate some of this core research into industrial applications. Research such as that detailed above has made significant advances into determining bearing failure as the root cause of malfunction. Detecting which bearing is failing across a complex system has received somewhat less research. Bearing prognostics is another area with much ongoing research being performedboth in the simulation and data-driven domains. To give an example, Hong et al. [20] combined grade life and extensive mathematical modeling techniques in order to produce prognostic models for aeroengine bearings. The results are described by the authors as "practical and verifiable." Although a number of similar recent studies exist, this work is of note for the extent of the studies performed which include bearing test stand run-to-failure validation. The lab results appear impressive; this research has yet to be applied and tested in real life applications-indicating that despite the number of parameters considered, it is still not possible to model naturally occurring phenomena sufficiently.

A large body of work in this area also exists from Borghesani et al. [21], where several examples of data driven techniques can be seen applied to a wide variety of bearing types. An example of recent developments involves the use of "cepstrum prewhitening" in order to remove sufficient noise for accurate bearing diagnosis and prognosis. This work is particularly noteworthy due to the emphasis on real-world applications, where the traditional lab-based techniques of order tracking and synchronous averaging do not provide sufficient noise removal for harsh industrial environments. The addition of such techniques is a crucial step in developing the current generation of diagnosis and prognosis algorithms for use in future IVHM systems.

2.6. Shaft Cracks. Another potentially serious fault in rotating machinery is shaft cracks, and so early detection of any such fault is highly important. Methods of crack formation and propagation can be diverse, and range from high and lowcycle fatigue to stress corrosion. Simulation and modeling of shaft cracks can have significant advantages over datadriven methods. Perhaps the most obvious advantage is the relative simplicity of inserting a fault into, for example, a finite element model as opposed to seeding a fault in a working industrial machine. As such, research into shaft cracks has been progressing steadily with the corresponding increases in computing power.

A clear synergy between data-driven and physics-based simulation research can be implied by a number of recent works of research. An example of recent advances from a data collection perspective is Li et al. [22], which details statistical models based on historical data for condition monitoring purposes. This unique work uses the human auditory system as inspiration for enriching methods of mechanical faults and features extraction. The results indicated by the paper are perhaps not as convincing as some other methods discussed in this paper, it describes an interesting "outside the box" method of tackling common problems.
From a modeling perspective, Bachschmid et al. [23] cover a wide range of vibration phenomena in order to develop a model-based identification and severity procedure. This work is noted for its thoroughness in modeling procedure, including accurate modeling of the crack breathing mechanism. A combination of high and low fidelity models is validated through experimental study, and "excellent" accuracy is claimed by the authors in detecting crack position and depth through the use of the proposed model-based diagnostics.

The nature of shaft cracks has resulted in a wide variety of research being performed into both localization and prognostics of these faults (indeed, the two topics can be considered related). Recent examples of work in this area include Inoue et al. [24], who detail crack localization using forced response modeling. In this case, a test rig was constructed consisting of a circular shaft supported by two bearings. Frequencydomain data was used to create a localization algorithm, designed in combination with an FE model. Although this research provides good accuracy of localization in a lab environment, it remains untested in a more complex system (e.g., a full gas turbine).

Sonnichsen [25] describes finite element modeling of crack propagation, with validation against experimental results provided to demonstrate the validity of such modeling techniques. The paper concentrates on natural frequencies and resonance curves. Whilst the authors claim improvements in the ability to understand such faults, again the system in question is quite simple - and such FEA models are difficult to scale up to full size applications.

2.7. Blade Cracks. Blade cracks, if allowed to develop, can result in serious consequences. Cracks can form due to high centrifugal stresses across operational cycles (in the case of an aircraft gas turbine, for example, start up, and take off through landing and taxi). As excessive crack growth can lead to catastrophic rotor/blade failure, early detection and prognosis of such faults are essential. As with shaft cracks, physics-driven simulation of blade cracks is an area of significant research. This varies from high-fidelity finite element models to low-fidelity system and mathematical models. The recent work demonstrated by Green and Casey [26] is a good example of recent mathematical modeling from a diagnosis perspective. In this paper, the authors concentrate on early detection using global and local asymmetry crack models. $2 \mathrm{X}$ harmonic components are identified as key areas for the early detection of blade cracks, however again this paper suffers from being applied and tested on a relatively simple system which may not scale up to a full size turbine.

Sonnichsen [25] demonstrated high-fidelity modeling, the authors used FEA to model crack growth, making comparisons and validating against an experimental rig. This work is particularly interesting as it outlines the advantages and drawbacks with the latest state-of-the-art modeling techniques.

Localization and prognosis of blade cracks have also benefitted from recent advances in simulation and modeling. Sawicki et al. [27] contains details of work on a novel active 
magnetic bearing system for use in the early detection, localization and prognosis of blade cracks. Again the emphasis is on early detection, with the bearings used to excite the system in order to obtain optimum response vibrations for analysis. The authors admit the approach has some merit in diagnosing blade cracks; however, it is in the early stages of development and work are ongoing. FEA has also been used extensively to support blade crack prognostic tools; Xiang et al. [28] are an extensive example of recent work. In this case, a number of advanced FEA methods are applied to produce accurate FEA solutions-these include surface-fitting techniques and the contour-plotting method. The authors experimentally validate their work and suggest that it can be applied to prognosis and quantitative diagnosis of blade cracks. Despite the claimed advances, again the scalability of such research to full size turbines is an issue-particularly with regard to the complexity of the FEA models.

2.8. Rotor Bow. Rotor bows can be a primary source of unwanted vibration in gas turbines. The main cause of a rotor bow (rotor bows do not include bows due to gravity) is thermal differences in a system caused by operating conditions. It is noted by Domes [5] that this nonsymmetrical thermal distribution can cause excessive unbalance to the extent where a gas turbine will not start correctly. Such rotor bows are common on start up or shut down, and are often accounted for in operational procedures. However, if thermal "hot spots" exceed a given tolerance level, they can cause permanent unbalances due to rotor deflections. Such rotor bows can lead to other faults, including rubbing and looseness which complicate isolation and localization.

Traditional data-driven techniques for detecting rotor bows involve combinations of slow roll and vibration data [29]. More recently, mathematical modeling techniques such as that detailed by Meagher et al. [30] have been used in order to diagnose residual rotor bows and differentiate these faults from other sources of unbalance. The authors of this paper build upon established methods for the models and are unique in that they concentrate on response at the bearing points. This is perhaps more useful for industrial applications, as vibration information is more readily available at bearing points rather than intrusive proximity probes, which are often used in lab work.

The little work that exists on attempting to localise rotor bows across complex systems tends to be data-driven in nature; see Gaka and Tabaszewski [31], where the authors used statistical symptoms based on known data as a method of diagnosing and prognosing a number of faults, including rotor bows and unbalance. This paper attempts to address the irregularities and fluctuations that occur over a long service life. In order to achieve this, a modified energy processor model is created, using data drawn from large steam turbines over long periods of life.

Prognosing rotor bows is a complex subject. As rotor bows are often caused by temperature deflections, making predictions for remaining useful life and potential future problems lies not only in the realm of mechanical rotordynamics but also to some extent in thermodynamics. The recent work detailed by Sinha [32] is of note for detailing diagnosis and quantification of various rotordynamic faults and describing the advantages of mathematical modeling over traditional vibration-based approaches. The topic of scalability regarding FEA models is discussed, including an argument for the use of partial (simplified) mathematical models for large, complex systems.

Another two works which are of interest with regard to modeling of rotor bows include Shen et al. [33], where the authors modeled a rotor-bearing system with a permanent rotor bow, looking at the impact of secondary faults such as rub. The study of fault combinations in this paper is useful for fault differentiation studies; however, the authors study a permanent, initial rotor bow. This therefore does not take into account developing or worsening faults and the different vibration phenomena that are observed as such faults are developing.

Lees et al. [34] describe the importance of model-based fault identification techniques and outline recent research in the area, providing a good reference paper for more research on this specific fault.

\section{Discussion}

3.1. Sensor Suites. The subject of sensor suites is of great importance with regard to future industrial applications. On one hand, advanced and complex sensor suites generally enable improved fault localization and diagnosis; however, the added complexity and cost has resulted in many of these systems being omitted from the latest generations of rotating machinery in industrial applications. From an industrial perspective, the cost benefit of additional sensors needs to be significant in order to justify this approach. In response, a number of works listed, including [8] as a good example, take the approach of achieving the same objectives using a greatly reduced sensor suite. In many ways, the required sensor suite for many of the currently researched fault diagnosis and prognosis techniques provides an indication of the ability of the techniques to be used practically in industrial situations. A complex suite may return a very high success rate on a lab-based rig; however, the impracticalities of mounting such suites on a real-world application negate the advantages.

Further to this, the consideration for sensor position must be considered. The use of proximity probes and keyphasor transducers has very clear advantages in a number of situations; however, the intrusive nature of the sensor prevents application in a number of complex systems. With this in mind, a number of the more promising studies listed rely on the simple suite of an accelerometer placed in positions which are relatively remote from the sources of vibration, potentially with a noisy transfer path.

3.2. Diagnosis. It can be seen from the research outlined in the previous section that the diagnosis of faults in rotating machinery is a subject of much ongoing research. This involves the improvement and development of traditional vibration monitoring techniques, development of new datadriven technologies, and novel research into physics-based 
simulation and modeling. In many cases, these topics of research are dependent on one another for reasons of validation, verification, and speed of analysis. In several cases, it can be seen that multiple faults have been modeled for the purposes of identification and isolation. However, no studies have yet been performed which deal with all of the aforementioned faults. All of these faults are intrinsically related to one another. Complex combinations of faults have begun to be analyzed with the emphasis on developing new diagnosis techniques. Physics-based modeling has proven to provide significant advances with regard to specific faults, notably shaft and blade cracks, where techniques such as FEA enable much easier, faster, and cheaper test data then seeding faults into live systems.

It can be noted, however, that both high and low-fidelity modeling techniques are being applied to cutting edge research for all of the listed rotordynamic faults (and others not detailed in this paper). In addition to the advantages in the speed of obtaining test results, physics-based simulation is providing another dimension to data-driven techniques. System models are being used as part of logic and reasoning suites in the identification and differentiation of various faults. High-fidelity models enable simulations of fault combinations, which are not possible practically or are prohibitively expensive in live systems.

Nevertheless, results obtained from modeling studies still need to be validated against proven data-driven techniques before implementation in industrial applications is possible. It is also of note from the literature reviewed for this paper that almost all modeling for the diagnosis of faults involves extensive simplification, often reducing potentially complex systems down to one or two disc/shaft/bearing models. Adapting the claimed results from such research to systems with many discs/bearings/shafts is important in improving existing rotating machinery diagnostic techniques.

The inclusion of nonlinear effects is notable amongst many authors-as the understanding of the complex faults and interactions continues to progress. Such research opens the potential for promising new avenues into areas of design for IVHM.

Finally, it is of note that despite the wide variety of advanced research here, a consensus for the most efficient techniques and algorithms has yet to emerge in order for the final adaption to industrial application to be achieved. However, the consideration for industrial application taken by many of the authors detailed in this paper provides promise for next generation IVHM systems.

3.3. Localisation. Fault localization in rotating machinery is an important topic of research for future condition-based monitoring systems. Knowing not only what type of fault has occurred, but also where in the system is an important consideration which can influence maintenance procedures in complex machinery. It is worth noting from the literature surveyed for this paper that many studies have focused on diagnosis and prognosis of single rotor/bearing systems. Often for legitimate reasons-simplification for computing speed, for example. Few studies, however, have taken into account the localization of faults across whole systems. One of the more extensive examples highlighted in this paper (Han et al. [15]) provides extensive analytical studies relevant to localization on a duel disk setup, utilizing Hilbert-Huang Transforms; however, in this example comprehensive validation is lacking, thus limiting the potential applicability of the research.

This problem is not limited to modeling and simulationbased research. Many newly developed data-driven techniques for diagnostics and prognostics claim good results by heavily instrumenting specific components of a test system. In many industrial cases, this is not possible, practical or costeffective.

To give some examples, a keyphasor transducer can be particularly useful in diagnosing faults such as rotor bow. However, this equipment requires the ability to cut a key way for measurements to be performed. Optical sensors have recently been applied to detect rotor unbalance; yet such a technology would be difficult to implement in a system with several rows of rotors (as in a gas turbine).

Whilst new diagnosis and prognosis techniques for faults in rotating machinery are being continuously researched, the lack of corresponding studies into localization can be considered one of the many challenges in promoting recent core research into live industrial applications.

3.4. Fault Differentiation. One promising development in recent modeling and simulation of rotordynamic faults is that of fault differentiation. Several researchers have moved on from studies on individual faults in order to concentrate on combinations of faults. As stated, rotordynamic faults such as those listed in this paper are linked to each other. Such studies, therefore, concentrate on such topics as a misalignment causing an unbalance or a looseness causing a rub. Findings from reports such as these are important in understanding complex anomalies. In industrial applications, simply detecting and rectifying an unbalance does not provide a satisfactory solution, if the root cause of the fault is a misalignment. This is a complex topic, as several faults can exhibit similar vibration characteristics, making traditional detection techniques inaccurate in some cases.

Making reference to some of the aforementioned studies, those such as Bachschmid et al. [23] and Sonnichsen [25] indicate some initial studies including several faults. The results of such studies, indicate a high success rate in differentiating the faults in the given conditions; however a number of limitations still exist. Studies such as these consider unbalance in the fault chains. This is typically "static" or simple unbalance applied to a single shaft/rotor-in reality unbalance faults can be more complex than this. In addition, the simple systems used to study and differentiate faults are very different from the complexities of a full turbine. However, further work may enable such research to "scaleup" to this stage with the aid of further validation and verification.

3.5. Prognosis. It can be seen from the examples of recent research described for common faults that prognostic techniques are a topic where much work is being performed. 
Some of this work would be very difficult or impossible to implement in an industrial situation; others provide promising results of use to future work in the area. Predicting the remaining useful life of components is critical in the development of condition-based monitoring strategies for industrial implementation.

It can be noted that prognostic studies for certain rotordynamic faults are considerably more advanced than for others. The most obvious examples of the faults detailed in this paper are bearing faults, shaft, and blade cracks. These are also areas where physics-driven simulations have had an important impact. The ability to design any fault type (or combinations of faults) into such simulations (to be its low-fidelity mathematical models or high-fidelity finite element analysis) has provided researchers with many different avenues to explore. Studies into the prognostics of other faults can be complicated by various factors. An unbalance, for example, has had relatively little research performed into prognosis. The fact that unbalance is often the cause of another underlying fault is one reason for the difficulty in researching prognosis in detail for this fault. The results of unbalance are also very dependent on the severity of the fault and the system in which it occurs.

It is worth noting that even for the faults where prognosis research is more advanced (e.g., shaft cracks); true development of the fault is not always studied. Fan et al. [17], for example, study sets of discrete cracks in order to "prognose" crack growth. In fact, this study is more akin to detecting fault severity with inferred effects on crack growth. In contrast, Li et al. [22] consider the continual development of such cracks; however, the authors do admit this work is in its infancy. Such points limit the ability for true prognosis to be achieved without extensive operational data.

It is also worth noting that combinations of faults have been simulated extensively for the purpose of diagnostics; however, few studies exist combining faults for the purpose of prognostics. Such studies are, however, a logical progression from some of those already performed. It may be that such prognostic studies build upon some of the condition-based maintenance (CBM) for diagnostics. High-level studies such as Jaw and Merrill [35] indicate how prognosis and diagnosis techniques can be combined into a CBM system. This study is designed for a military aeroengine, indicating the desire for such systems to be implemented-although the architecture described has the capability of including various algorithms for fault prognosis, the amount of consideration for prognosis of fault combinations is unclear.

3.6. Modelling. The large number of works reviewed for this paper which includes modeling techniques in order to aid diagnosis and prognosis indicates the possibilities provided by modern computer power and software development. Broadly, the modeling studied in the aforementioned papers breaks down into two categories: mathematical modeling and finite element analysis (FEA). The mathematical models, such as Borghesani et al. [21], often provide a theoretical basis upon which data driven or FEA studies can build and validate. Mathematical models, despite being a traditional approach to rotordynamic problems, therefore continue to be developed to tackle more advanced problems.

FEA techniques offer a constantly expanding area of simulation to explore. They are used for research as diverse as crack propagation to unbalance localization [36]. Modern codes such as NASTRAN and Ansys enable rotordynamics to be studied beyond the traditional stress and modal analysis. The ability to study the effects of rotation (e.g., through Campbell diagrams and transient analysis) continues to drive research and innovation in this area. The studies discussed in this paper indicate the power of FEA, with some highly accurate simulations having been performed. However, there is still a limitation of computer power-applying some of the FEA techniques to whole engine models would prove to be too computationally expensive to be viable. As a result, alternate methods such as model order reduction and system level modeling (and model-based reasoning for implementation) are still required in order to make some of the FEA studies viable in an industrial environment.

Nonlinearities continue to form an important part of many recent studies, with the complexity and detail of the specified faults being continually expanded. The depth of modeling has enabled in many cases accurate validation against experimental approaches. Despite this, complete understanding of the vibrational phenomena of rotating machinery is not currently possible, despite the drive towards this end.

3.7. Rotordynamics and IVHM. The bulk of current research into rotordynamics from the point of view of prognostic health management (PHM) can be roughly divided into two types: initial single-fault diagnosis/prognosis techniques and studies into the general requirements and limitations of current systems along with current and future trends. An example of the latter is Pusey [37] who provides a good summary overview of current diagnosis and prognosis techniques with regard to condition-based maintenance.

As a result of this split, a clear gap exists between the core research being performed into rotordynamics from a condition-based maintenance perspective and the identified needs of industry. Taking a fledgling piece of research and applying it to a commercially-ready system (e.g., a gas turbine engine for an aircraft) is a long and complex task. It is nevertheless worth noting that technologies for automatically detecting an unbalance or misalignment in a gas turbine were developed over 10 years before the latest commercial aircraft were conceptualized, and yet these planes are still limited in this capacity. This highlights the need for work which links the fundamental research into individual fault diagnosis to "live systems" in use in industry.

Physics-based simulation and modeling of rotordynamic parts is a well-researched field. Such modeling has been used as the basis of diagnosis and prognosis of faults by many researchers; several recent examples have been outlined in this paper. Occasional pieces of work have been performed into modeling multiple faults, such as Jain and Kundra [38] who use a system model for online identification of unbalance and cracks. Beyond this, however, very limited research 


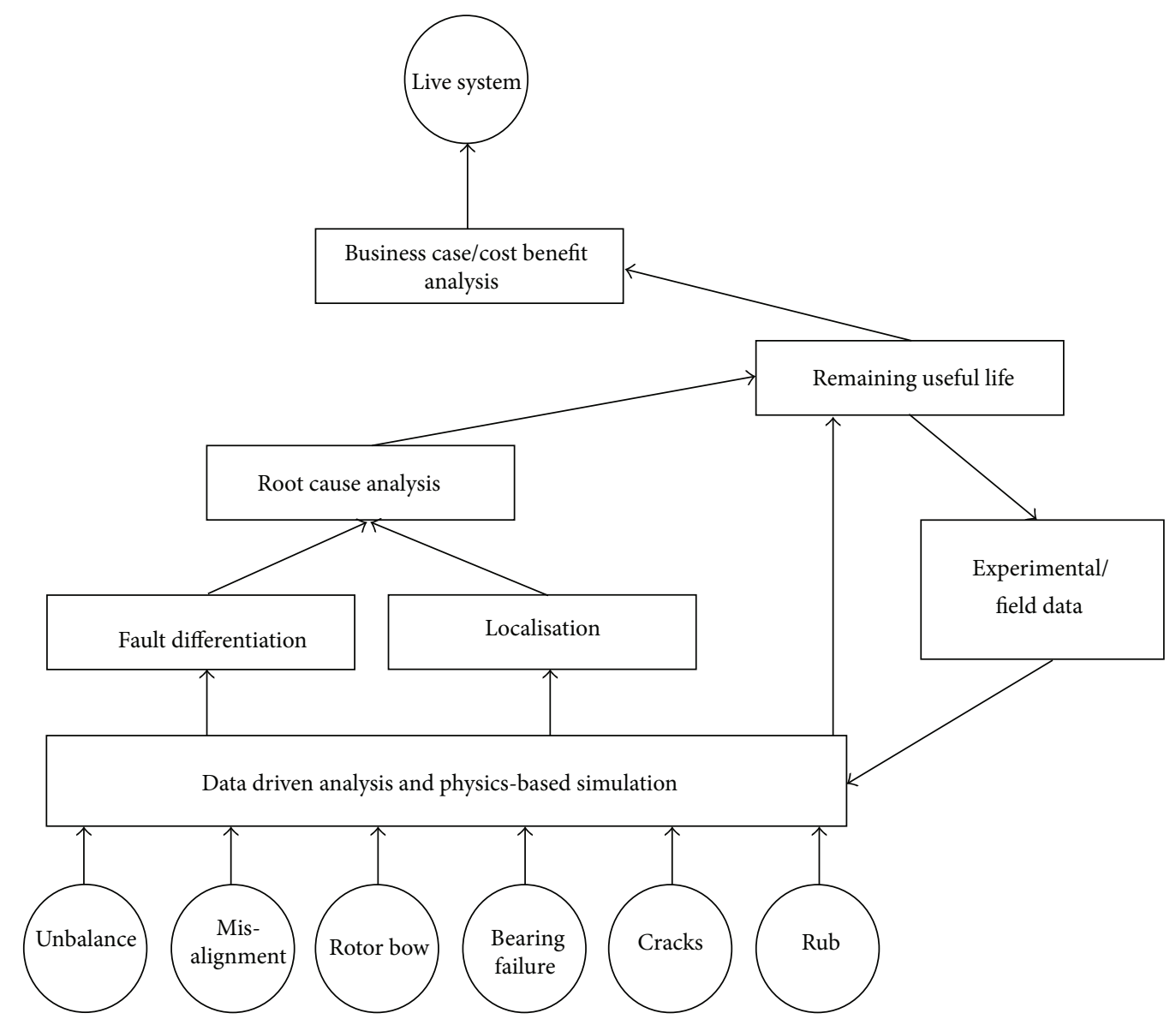

FIgure 1: Physics-based simulation-from research to industry.

exists. The demands of PHM techniques in industry are such that any system must not only be capable of detecting multiple faults, but must also be capable of detecting these faults across a range of different systems. Other considerations include the aforementioned ability to differentiate between multiple faults. Processing also needs to be taken into account, as the objective of these systems is to implement efficient condition monitoring and condition-based maintenance procedures. If processing data is a long, power-hungry process, then this aim cannot be achieved.

Figure 1 details a potential framework required in order to push core research, such as that detailed in this report, towards industrial applications. Many studies now exist on individual rotordynamic faults across a wide range of conditions and applications. Some studies have taken this further, with advanced prognostic models and diagnosis of dual faults (primary cause and secondary effect). Future research in the area of rotordynamics from a PHM perspective could potentially provide the bridge between these studies and live systems, by combining physics-based simulations with datadriven techniques and validating against experimental data.

Although quantifying the success of the research studied is difficult, it is possible to define the key areas in which a technique must excel in order to be considered viable. The work by Wheeler et al. [39] discusses in detail metrics for diagnostic and prognostic analysis, as does that by Vachtsevanos [40] and Saxena et al. [41]. The conclusions drawn from these papers and applied in practice to research like that covered by this paper indicate that the following metrics are important when considering the potential of a given technique to diagnose faults: coverage, false positive rate, and false negative rate. In the case of prognosis, probabilities, and lead time to failure are other important considerations. This criteria enables research to be assessed in terms of its suitability for industrial applications. Unfortunately, information on these metrics is not made readily available by the authors of most papers.

In terms of evaluating the effectiveness of the research discussed in this paper, there are difficulties in recommending a specific technique over others for general application. Most papers reviewed for this document (not just those referenced and discussed in detail) take a technique (new or evolved), validate for a given, specific system, and report success of the research. Despite this, some conclusions can be drawn from assessing common techniques applied across different studies and different faults. Although it is not possible to define the most common methods for diagnosing and prognosing faults in terms of numbers (as it was not possible to cover all recent 
rotordynamics research for this paper), as perceived by the authors, the following techniques have featured prominently in the reviewed research.

(i) Sensors: accelerometers.

(ii) Theoretical studies: mathematical modeling.

(iii) Physics-based simulation: FEA.

(iv) Data-driven: joint time/frequency domain analysis.

(v) Implementation: neural networking.

These techniques appear to be among the most promising currently under development, as they tend to feature numerous times amongst some of the work with wider scope, across fault types and with the most comprehensive validation. There are of course many subsections to these techniques; however, it shows one general direction of research and the clear possibilities posed in these areas.

Future developments in the field of IVHM for rotating machinery may incorporate these techniques alongside extensive use of nonlinear modeling and multiple fault interactions. The field of design for IVHM has only recently emerged; however, the potential exists for specific nonlinearities to be designed into a system in order to enable accurate diagnosis and prognosis of faults. The development of current algorithms to include diagnosis, localization, and prognosis of a range of faults will provide a significant advancement for future generations of IVHM systems. This combined with cost-effective sensor suites indicates the potential for evolutions of some of the research detailed here to form part of next generation IVHM suites for rotating machinery.

\section{Conclusion}

This paper has reviewed some of the latest research around a number of rotordynamic faults, namely unbalance, misalignment, rub and looseness, fluid-induced instability, bearing faults, shaft cracks, blade cracks, and rotor bow. Each fault was reviewed from the perspective of sensors, diagnosis, prognosis, localization, and modeling.

Key examples of recent work into the eight described faults have been detailed through works by a number of eminent authors. Additional work has been summarized and formatted for easy reference. Some current trends amongst the recent body of work include developments in the vast area of modeling nonlinearities, combinations of high and low fidelity modeling, and synergy between data driven and physics-based simulation approaches.

Despite the large volume of promising research reviewed, further development in a number of areas is required in order to produce effective next generation IVHM systems. As such, future developments may include fusion of and/or development of current algorithms to encompass all eight faults detailed, consideration of prognosis, diagnosis, and localization achieved using a reduced, cost-effective sensor suite.

\section{References}

[1] I. K. Jennions, Integrated Vehicle Health Management: Perspectives on an Emerging Field, 2011.
[2] G. Anderson, "Providing best-value IVHM solutions for aging aircraft," in Proceedings of the 9th Joint FAA/DoD/NASA Aging Aircraft Conference, vol. 1, pp. 1-11, 2005.

[3] A. Muszynska, Rotordynamics, Taylor \& Francis, 1st ed edition, 2005.

[4] D. E. Bently, Fundimentals of Rotating Machinery Diagnostics, ASME Press, 1st edition, 2002.

[5] B. Domes, "Vibration phenomena in aero-engines," in Proceedings of the 9th International Conference on Vibrations in Rotating Machinery Exeter (IMechE '08), vol. 1, pp. 15-32, September 2008.

[6] A. Hess, G. Calvello, and T. Dabney, "PHM a key enabler for the JSF autonomie logistics support concept," in Proceedings of the IEEE Aerospace Conference Proceedings, pp. 3543-4549, March 2004.

[7] S. N. Ganeriwala, B. Schwarz, and M. H. Richardson, "Operating deflection shapes detect unbalance in rotating equipment," Sound and Vibration, vol. 43, no. 5, pp. 11-13, 2009.

[8] G. N. D. S. Sudhakar and A. S. Sekhar, "Identification of unbalance in a rotor bearing system," Journal of Sound and Vibration, vol. 330, no. 10, pp. 2299-2313, 2011.

[9] T. Yang and M. W. Hsu, "An efficient diagnosis technique for variations of shaft-bow and unbalance," in Proceedings of the ASME International Design Engineering Technical Conferences and Computers and Information in Engineering Conference (DETC '09), pp. 57-66, San Diego, Calif, USA, September 2009.

[10] A. El-Shafei, S. H. Tawfick, and M. O. A. Mokhtar, "Experimental investigation of the effect of angular misalignemt on the instability of plain journal bearings," in Proceedings of the ASME/STLE International Joint Tribology Conference (IJTC'09), pp. 171-173, Memphis, Tenn, USA, October 2009.

[11] A. El-Shafei, S. H. Tawfick, and M. O. A. Mokhtar, "Misalignment modeling in rotating systems," in Proceedings of the ASME Turbo Expo, H. Bahaloo, A. Ebrahimi, and M. Samadi, Eds., vol. 1711, pp. 973-979, Orlando, Fla, USA, June 2009.

[12] L. F. Villa et al., "Statistical diagnosis based on vibration analysis for gear test-bench under non-stationary conditions of speed and load," Mechanical Systems and Signal Processing, vol. 29, pp. 436-446, 2012.

[13] C. F. Ngolah et al., "Intelligent fault recognition and diagnosis for rotating machines using neural networks," Software Science and Computational Intelligence, vol. 3, no. 4, 17 pages, 2011.

[14] Y. Lu, Z. Ren, H. Chen, N. Song, and B. Wen, "Study on looseness and impact-rub coupling faults of a vertical dual-disk cantilever rotor- Bearing system," Key Engineering Materials, vol. 353-358, no. 4, pp. 2479-2482, 2007.

[15] Q. Han, Z. Zhang, and B. Wen, "Periodic motions of a dualdisc rotor system with rub-impact at fixed limiter," Proceedings of the Institution of Mechanical Engineers, vol. 222, no. 10, pp. 1935-1946, 2008.

[16] H. F. de Castro, K. L. Cavalca, and R. Nordmann, "Whirl and whip instabilities in rotor-bearing system considering a nonlinear force model," Journal of Sound and Vibration, vol. 317, no. 1-2, pp. 273-293, 2008.

[17] C. C. Fan et al., "Mechanical systems and signal processing," Study of Start-Up Vibration Response for Oil Whirl and Dry Whip, vol. 25, pp. 3102-3115, 2011.

[18] K. Kappaganthu and C. Nataraj, "Nonlinear modeling and analysis of a rolling element bearing with a clearance," Communications in Nonlinear Science and Numerical Simulation, vol. 16, no. 10, pp. 4134-4145, 2011. 
[19] T. C. Gupta, K. Gupta, and D. K. Sehgal, "Instability and chaos of a flexible rotor ball bearing system: an investigation on the influence of rotating imbalance and bearing clearance," Journal of Engineering for Gas Turbines and Power, vol. 133, no. 8, Article ID 082501, 11 pages, 2011.

[20] J. Hong, X. Miao, L. Han, and Y. Ma, "Prognostics model for predicting aero-engine bearing grade-life," in Proceedings of the ASME Turbo Expo, vol. 1, pp. 639-647, Orlando, Fla, USA, June 2009.

[21] P. Borghesani, P. Pennacchi, R. B. Randall, N. Sawalhi, and R. Ricci, "Application of cepstrum pre-whitening for the diagnosis of bearing faults under variable speed conditions," Mechanical Systems and Signal Processing, 2012.

[22] Y. Li, J. Zhang, L. Dai, Z. Zhang, and J. Liu, "Auditory-modelbased feature extraction method for mechanical faults diagnosis," Chinese Journal of Mechanical Engineering (English Edition), vol. 23, no. 3, pp. 391-397, 2010.

[23] N. Bachschmid, P. Pennacchi, and E. Tanzi, "Cracked rotating shafts: typical behaviors, modeling and diagnosis," IUTAM Symposium on Emerging Trends in Rotor Dynamics, vol. 1011, pp. 441-454, 2011.

[24] T. Inoue, N. Nagata, and Y. Ishida, "Fem modelling and experimental verification of a rotor system with a open crack," in Proceedings of the ASME International Design Engineering Technical Conferences and Computers and Information in Engineering Conference (DETC '09), pp. 1113-1122, San Diego, Calif, USA, September 2009.

[25] H. E. Sonnichsen, "Real-time detection of developing cracks in jet engine rotors," in Proceedings of the IEEE Aerospace Conference, pp. 173-184, March 2000.

[26] I. Green and C. Casey, "Crack detection in a rotor dynamic system by vibration monitoring-part I: analysis," Journal of Engineering for Gas Turbines and Power, vol. 127, no. 2, pp. 425436, 2005.

[27] J. T. Sawicki, M. I. Friswell, A. H. Pesch, and A. Wroblewski, "Condition monitoring of rotor using active magnetic actuator," in Proceedings of the ASME Turbo Expo, pp. 1257-1265, Berlin, Germany, June 2008.

[28] J. Xiang, X. Chen, Q. Mo, and Z. He, "Identification of crack in a rotor system based on wavelet finite element method," Finite Elements in Analysis and Design, vol. 43, no. 14, pp. 1068-1081, 2007.

[29] M. G. Maalouf, "Slow speed vibration signal analysis: if you can't do it slow, you can't do it fast," in Proceedings of the ASME Turbo Expo, pp. 559-567, Montreal, Canada, May 2007.

[30] J. Meagher, X. Wu, and C. Lencioni, "Response of a warped flexible rotor with a fluid bearing," International Journal of Rotating Machinery, vol. 2008, Article ID 147653, 9 pages, 2008.

[31] T. Gaka and M. Tabaszewski, "An application of statistical symptoms in machine condition diagnostics," Mechanical Systems and Signal Processing, vol. 25, no. 1, pp. 253-265, 2011.

[32] J. K. Sinha, "Recent trands in fault quantification in rotating machines," Advances in Vibration EngIneerIng, vol. 8, no. 1, pp. 79-85, 2009.

[33] X. Shen, J. Jia, and M. Zhao, "Nonlinear analysis of a rub-impact rotor-bearing system with initial permanent rotor bow," Archive of Applied Mechanics, vol. 78, no. 3, pp. 225-240, 2008.

[34] A. W. Lees, J. K. Sinha, and M. I. Friswell, "Model-based identification of rotating machines," Mechanical Systems and Signal Processing, vol. 23, no. 6, pp. 1884-1893, 2009.
[35] L. C. Jaw and W. Merrill, "CBM+ research environmentfacilitating technology development, experimentation, and maturation," in Proceedings of the IEEE Aerospace Conference (AC '08), March 2008.

[36] R. Walker, S. Perinpanayagam, and I. K. Jennions, "Simulating Unbalance for Future IVHM Applications," in Proceedings of the Society for Experimental Mechanics (IMAC '12), vol. 29, pp. 141148, May 2012.

[37] H. C. Pusey, "Turbomachinery condition monitoring and failure prognosis," Sound and Vibration, vol. 41, no. 3, pp. 10-15, 2007.

[38] J. R. Jain and T. K. Kundra, "Model based online diagnosis of unbalance and transverse fatigue crack in rotor systems," Mechanics Research Communications, vol. 31, no. 5, pp. 557-568, 2004.

[39] K. R. Wheeler, T. Kurtoglu, and S. D. Poll, "A survey of health management user objectives related to diagnostic and prognostic metrics," in Proceedings of the 29th Computers and Information Engineering Conference, vol. 2, pp. 1287-1298, 2009.

[40] G. Vachtsevanos, "Performance metrics for fault prognosis of complex systems," in IEEE Systems Readiness Technology Conference (AUTOTESTCON '03), pp. 341-345, September 2003.

[41] A. Saxena, J. Celaya, E. Balaban et al., "Metrics for evaluating performance of prognostic techniques," in Proceedings of the International Conference on Prognostics and Health Management (PHM '08), pp. 1-17, October 2008. 

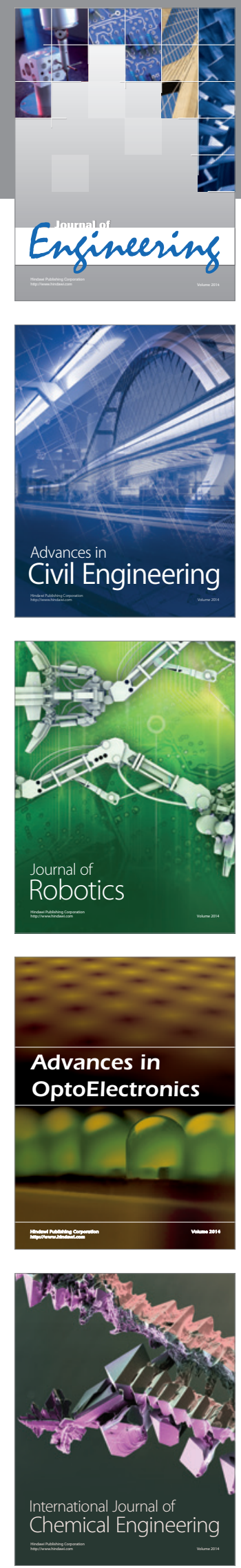

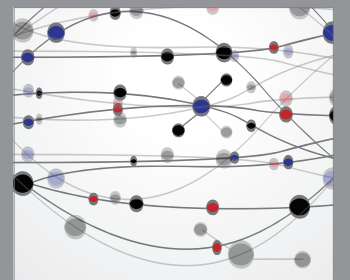

The Scientific World Journal
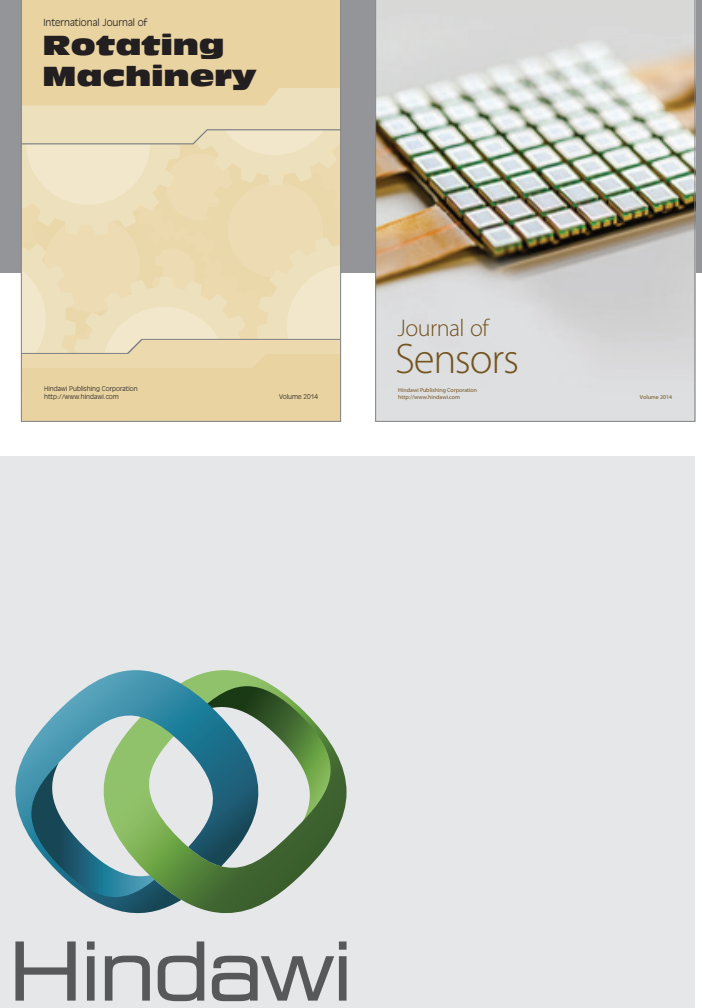

Submit your manuscripts at http://www.hindawi.com
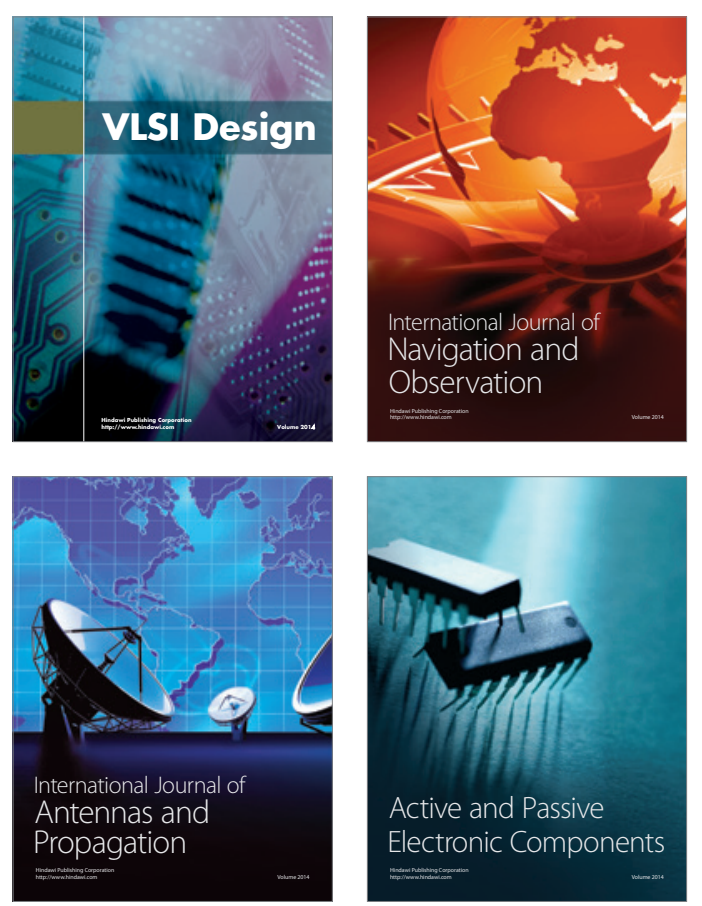
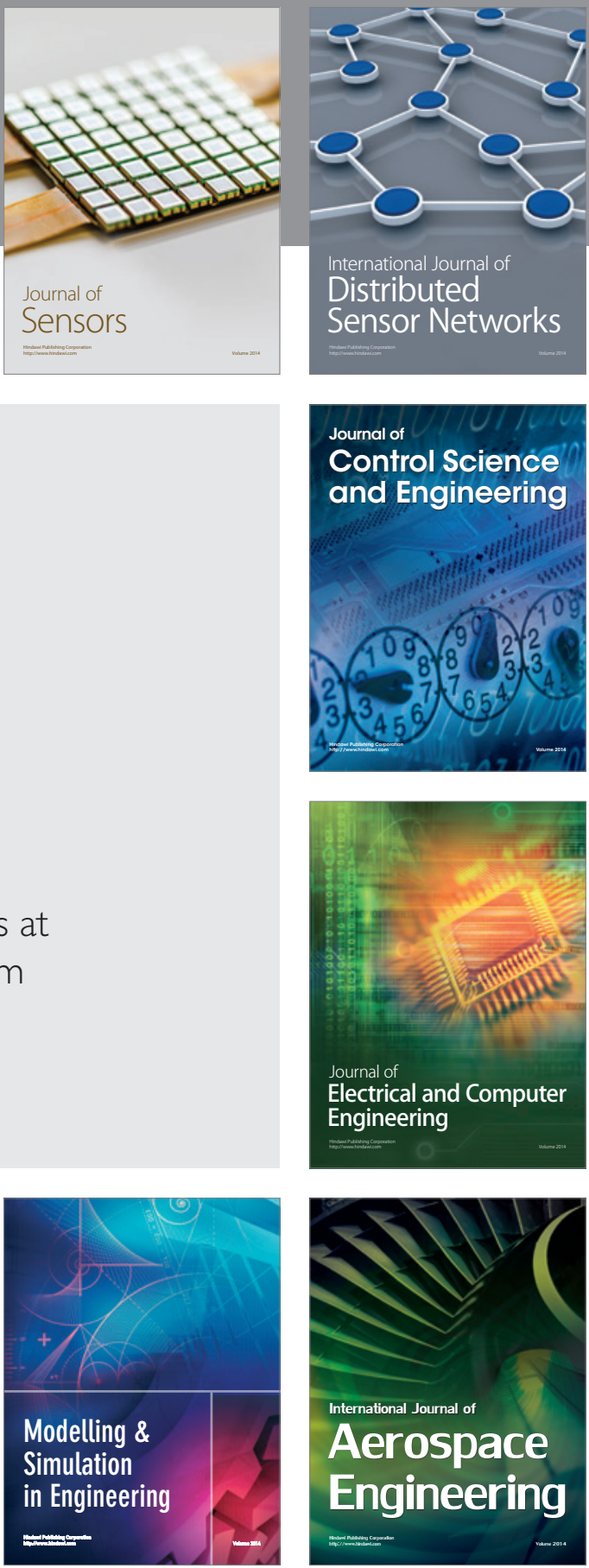

Journal of

Control Science

and Engineering
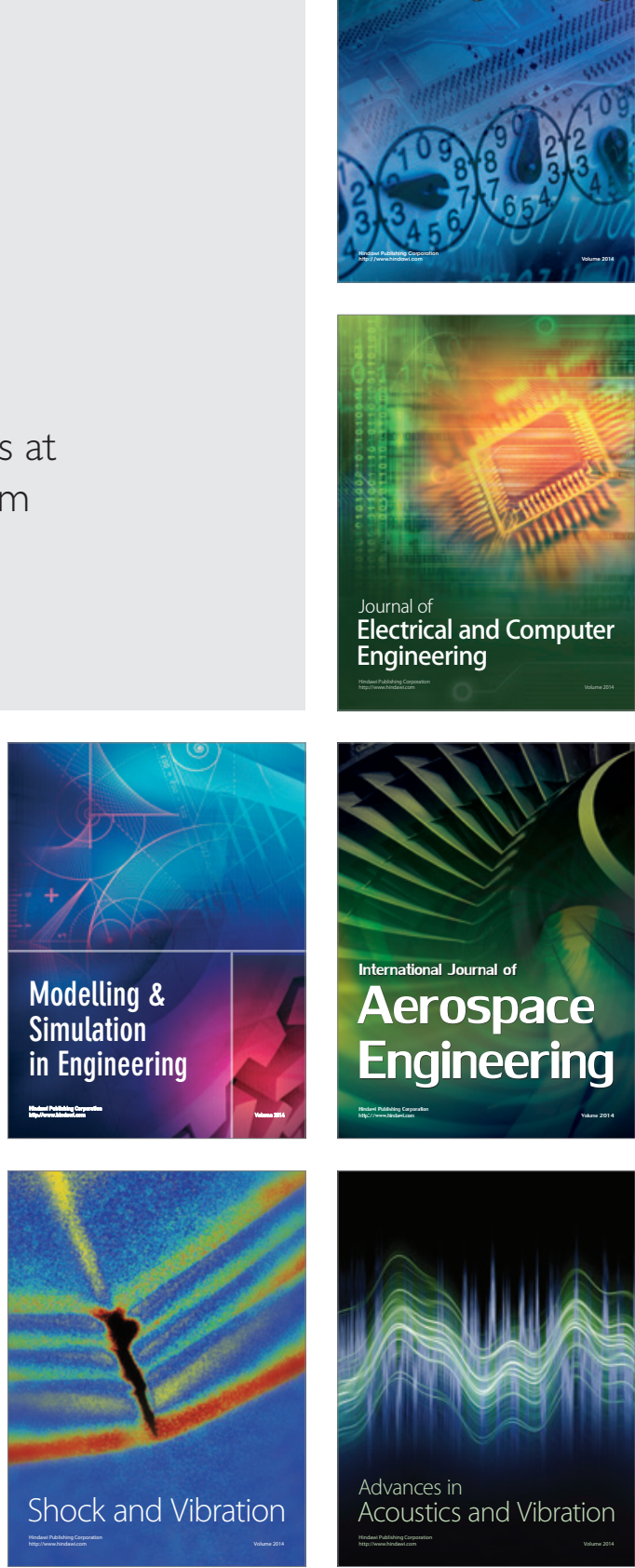